
internationales

vol. 32 - $n^{\circ} 1 \mid 2016$

Les migrations des Roms roumains en Europe

\title{
Les Roms roumains en région parisienne : les mobilisations associatives au prisme des temporalités migratoires et de l'habitat
}

Romanian Roma in Paris: Activities Associated to Migratory Temporalities and Housing

Los gitanos rumanos en la región parisina: movilizaciones asociativas bajo el prisma de las temporalidades migratorias y de la vivienda

\section{Céline Bergeon}

\section{(2) OpenEdition}

\section{Journals}

Édition électronique

URL : https://journals.openedition.org/remi/7674

DOI : 10.4000/remi.7674

ISSN : $1777-5418$

Éditeur

Université de Poitiers

Édition imprimée

Date de publication : 1 mars 2016

Pagination : 101-122

ISBN : 979-10-90426-27-6

ISSN : 0765-0752

Référence électronique

Céline Bergeon, « Les Roms roumains en région parisienne : les mobilisations associatives au prisme des temporalités migratoires et de l'habitat », Revue européenne des migrations internationales [En ligne], vol. 32 - $n^{\circ} 1$ | 2016, mis en ligne le 01 mars 2018, consulté le 14 avril 2022. URL : http:// journals.openedition.org/remi/7674; DOI : https://doi.org/10.4000/remi.7674 


\section{Les Roms roumains en région parisienne : les mobilisations associatives au prisme des temporalités migratoires et de l'habitat}

\section{Céline Bergeon ${ }^{1}$}

"Quand tu arrives ici à Paris, tu es étranger... et tu es Rom "

(Brishen, entretien réalisé à Paris en 2012).

Sur la scène européenne, Paris et sa région constituent une destination privilégiée par les Roms migrants en provenance de Roumanie. L'adhésion de la Roumanie à l'Union européenne, en 2007, a permis aux ressortissants roumains de bénéficier de conditions de circulation assouplies. Les déplacements des Roms, première minorité ethnique en Roumanie, s'inscrivent dans ce contexte de mobilité ce qui provoque, depuis plusieurs années, de nombreux débats et textes réglementaires ${ }^{2}$, tant à l'échelle des nations roumaine et française qu'à celle des instances européennes. Ces Roms migrants sont estimés entre 15000 et 20000 personnes en France ${ }^{3}$. Si l'expression renvoie explicitement à leur statut de migrant, c'est notamment pour les distinguer d'autres populations établies de longue date en France - les Tsiganes ${ }^{4}$ français (Olivera, 2009) - qui sont pour la majeure partie d'entre eux des citoyens français, soumis aux conditions de circulation posées par le droit interne; ce qui n'est pas le cas des familles roumaines de culture rom qui circulent notamment depuis la chute des régimes communistes des pays de l'Est vers l'Europe de l'Ouest - et qui sont, de fait, soumis au régime des politiques migratoires internationales. Même si I'on constate un point commun entre lesTsiganes français et les Roms roumains

\footnotetext{
1 Maître de conférences en géographie, Université de Poitiers, MIGRINTER (UMR 7301), MSHS, bât. A5, 5 rue Théodore Lefebvre, TSA 21103, 86073 Poitiers cedex 9 ; celine.bergeon@univ-poitiers.fr

2 À titre d'exemple, ils ont été, malgré eux, à l'origine des premiers accords de réadmission entre la France et la Roumanie en 1992 (Bergeon, 2010 : 203).

3 Source : Romeurope.

4 Dans les recherches anthropologiques et sociologiques, le terme de "Tsigane " (ou "Tzigane "), rassemble quatre groupes distincts ; celui des Manouches, des Gitans, des Yéniches et celui des Roms. En France, la catégorie administrative " Gens du voyage " représente les citoyens français se reconnaissant gitans, manouches, yéniches ou rom. Bien que pour la plupart, ils soient sédentarisés, des familles ont encore un mode de vie circulant. Ces familles possèdent des papiers d'identité français et leur présence en France remonte pour la plupart d'entre eux au Xle siècle.
} 
migrants en France - celui d'un accueil très souvent inhospitalier - I'amalgame reste tenace dans les discours politiques, I'opinion publique et au sein même de la nébuleuse associative. "Réels étrangers de l'intérieur" (Robert, 2007) pour les Tsiganes français, " minorité problématique " (Olivera, 2009) pour les Roms de l'Europe de l'Est, leur présence est montrée comme un problème social majeur. La catégorie rom migrant, " chimère ethno-juridique " (Coquio et Poueyto, 2014 : 27) est aujourd'hui validée et utilisée par les instances juridiques nationales et européennes comme s'il existait une " migration rom " spécifique, parmi celles opérées par des migrants en provenance d'autres parties du monde qui souhaitent rejoindre l'Europe. Cette " question rom " est donc un objet difficile à saisir et s'établit, en réalité, " dans des jeux de langages " (Legros et Rossetto, 2011) qui s'opèrent dans la sphère politique et médiatique. Si la catégorie " rom migrant " a le défaut de posséder une connotation ethnique - pourquoi ne pas simplement parler de Roumains ? - elle, pose, malgré tout, le contexte juridique dans lesquels s'effectuent les déplacements, celui de la migration.

En France, plus particulièrement dans les grandes agglomérations ( $\mathrm{Nacu}$, 2010 : 141), la visibilité de leur présence, appuyée par une importante couverture médiatique, s'opère notamment à travers des actions de démantèlements de squats ou de campements illégaux, des opérations d'expulsions massives et de retours forcés vers la Roumanie. Au-delà d'une réalité qui pose problème aux autorités, la présence des Roms ne peut se résumer aux situations de conflit engendrées par leurs installations souvent illégales. L'investissement de l'espace public se fait aussi de manière inédite, par le biais de manifestations culturelles peu ou très visibles qui participent, malgré tout, à la construction d'une légitimité sociale (Cordeiro et Hily, 2000 : 59-76) très souvent remise en cause lorsqu'il s'agit des Roms.

À partir de deux entrées, celles des temporalités migratoires et des types d'habitat, nous verrons, dans cet article, comment se décline la participation aux événements culturels et comment se construisent les revendications identitaires en région parisienne. Nous nous interrogerons sur l'impact de ces " temps migratoires " sur la force ou la faiblesse des liens qui se développent à plusieurs échelles : au niveau local avec les habitants, les voisins et au niveau transnational avec les membres de la famille restés en Roumanie ou dispersés dans d'autres pays européens.

Nous présenterons, dans un premier temps, les aspects de cette migration rom qui occupe, depuis maintenant quelques années, les discours politiques. Ce point nous permettra ainsi d'alimenter les réflexions autour du transnationalisme et des réseaux migratoires et de déconstruire une image stéréotypée de la migration rom. Cela nous permettra également d'entrevoir des changements concernant cette migration, notamment celui du rôle de la femme dans le processus migratoire. Dans un second temps, nous présenterons le paysage associatif en direction des Roms en région parisienne. Ces associations sont les premiers médiateurs entre les familles roms, la population locale et les institutions politiques. Nous considérons ces structures comme des outils pertinents pour mesurer la participation (dans sa nature, sa fréquence, etc.) des Roms roumains dans les différentes manifestations culturelles ainsi que les retombées de ces manifestations pour les familles. Un des enjeux de cette seconde partie est de souligner cette activité associative et de montrer les capacités des familles 
à participer à la vie de la cité malgré un accueil parfois fortement négatif. Enfin, à la lumière des propos recueillis auprès des familles, nous verrons dans une troisième partie l'impact des temporalités migratoires et des modes d'habitat sur l'implication des Roms dans les manifestations culturelles; ce qui nous permettra de voir aussi qu'à partir des " moments difficiles de la migration " (conditions de vie, expulsion, image dépréciative, etc.) émergent des forces et des investissements humains particuliers.

Pour nourrir cette réflexion, des données issues d'une recherche ${ }^{5}$ initiée depuis 2011 auprès de migrants internationaux vivant en squats et en campements illégaux sont mobilisées. Le cœur de ce travail est consacré à l'analyse des conditions de vie des migrants et de leurs sociabilités quotidiennes dans leurs quartiers et plus globalement dans la ville. Parmi l'ensemble des migrants rencontrés ${ }^{6}$, une grande partie d'entre eux sont des Roumains de culture rom ; ils sont originaires, pour la plupart, des Judeţe (département) d'Arad, Bihor, Constanta et Timis. Les conditions de vie de ces migrants étant parfois très précaires (absence d'eau, d'électricité, menace de l'expulsion, etc.), des entretiens formels ont été difficilement organisables. C'est plus précisément au cours de discussions autour d'un café ou d'un repas que les informations ont circulé. Aux vingt-sept entretiens ${ }^{7}$ menés au total, quinze d'entre eux ont été réalisés auprès de Roms, tant sur le lieu de vie (chambre et salon dans les squats et à l'intérieur des baraques pour les campements) que dans des lieux délocalisés dans le quartier ou plus éloignés dans la ville (bars et parcs). Tout en considérant ces échanges informels de grande qualité, des récits de vie et des entretiens biographiques ont aussi été recueillis auprès de familles avec lesquelles des liens de proximité et de confiance ont pu être instaurés dans la durée. Pendant les réunions et les moments de fête, l'observation a été privilégiée, facilitée par du bénévolat au sein de deux associations (la CIMADE et Romeurope). Enfin, ce matériau a été croisé avec des entretiens auprès d'acteurs associatifs afin de comprendre le dialogue entre migrants et acteurs associatifs.

\footnotetext{
5 Recherche menée à Paris, Barcelone, Rennes et Poitiers.

6 Les squats et les campements accueillent des profils très diversifiés de personnes migrant seules ou en famille. Au total, dix-sept lieux de vie illégaux ont été investis depuis 2011 et suivis sur toute leur durée de vie, ce qui a notamment permis de déceler des logiques d'appropriation de l'espace et de pratiques quotidiennes spécifiques (Bergeon et Hoyez, 2015). Certains de ces lieux sont toujours en activité, d'autres ont été démantelés. Parmi ces migrants, les nationalités les plus représentées sont les Géorgiens, les Albanais, les Mongols, les Congolais, les Sénégalais, les Mauritaniens, les Nigérians et les Roumains.

7 Les grilles d'entretien sont constituées de trois axes : la trajectoire résidentielle et migratoire, I'appropriation de l'espace de vie et du chez soi en contexte résidentiel précaire, et les sociabilités externes au lieu de vie (ensemble des activités réalisées dans le quartier et la ville et leurs contenus). C'est notamment dans le cadre de ces deux derniers axes que l'importance des manifestations culturelles organisées en dehors et à I'intérieur de l'espace de vie a été soulignée par les familles.
} 


\section{Des parcours migratoires diversifiés s'inscrivant dans un transnationalisme familial}

\section{Circulation, famille et réseaux de parenté}

Les déplacements des Roms se caractérisent par un aspect circulatoire (Marušiakova et Popov, 2008 ; Bergeon, 2011) qui ne leur est pas spécifique, les migrations roumaines, en général, s'inscrivent dans ce schéma d'allers et retours réguliers au pays (Diminescu, 2001 ; Potot, 2007) et ne se distinguent pas, de façon plus générale, des déplacements opérés depuis l'Europe de l'Est et les Balkans (Olivera, 2009) par d'autres migrants. La diversité des parcours migratoires recueillis a permis de démystifier ces migrations roms souvent perçues comme issues d'une " culture nomade ". Ainsi, il n'existe pas de modèle stéréotypé de la migration rom.

Si les motivations économiques sont un des premiers facteurs de migration, les migrants roms roumains fuient également un environnement social fortement discriminant (Reyniers, 1995 ; Pons, 1995). En effet, une grande partie d'entre eux subissent une situation sociale et politique très précaire en Roumanie (Delépine, 2007 ; Guest et Nacu, 2008). À cela s'ajoute une marginalité économique très marquée et accentuée par les changements socio-politiques opérés depuis 1990. Les Roms, dépourvus de bien fonciers et immobiliers n'ont pas pu profiter des redistributions et de la privatisation des biens nationalisés (Guest et Nacu, 2008) opérés tout au long des années 1990. Cette précarité économique se traduit aujourd'hui par un taux de chômage élevé pour les Roms en Roumanie, leur laissant ainsi comme principale alternative des activités caractérisées par l'informalité. La précarité économique est le facteur déclenchant de ces déplacements, ce qui ne les singularise pas d'autres mouvements migratoires. Par ailleurs, la structuration et le maintien des réseaux en migration se déclinent très largement dans l'entourage familial et les réseaux de parenté (Reyniers, 2008). Cette proximité familiale - et les solidarités qui en découlent - fait l'objet d'une forte visibilité, notamment à l'échelle locale, dans le cadre des pratiques de logement, qu'elles soient informelles ou légales. Ces familles élargies, qui partagent le quotidien de la migration, proposent une organisation familiale qui disparaît au profit du modèle nucléaire qui caractérise les sociétés actuelles. Dans le cas des familles roms, et dans la lignée des travaux de Todd (1994), la structure familiale joue un rôle fondamental sur les comportements et les logiques migratoires. En effet, le " socle migratoire " s'établit à partir de la dispersion de la parenté au sein de l'Europe ainsi qu'à travers les liens étroits, parfois très soutenus, avec le village d'origine en Roumanie. Les différents lieux d'installation permettent la circulation des activités, des biens et des " apprentissages migratoires " par le biais d'une mobilité tissée "par-dessus les frontières " (Fibbi et D'Amato, 2008) et construisant de réels espaces transnationaux. Faisant directement écho aux réflexions menées sur le transnationalisme depuis les années 1990, les migrations des Roms roumains s'établissent dans des réseaux économiques, sociaux et familiaux qui sont liés entre eux par des relations proches ou plus distendues selon les " moments migratoires". Nous nous situons ainsi dans la lignée des travaux menés par Bash et al. (1994) qui décrivent le transnationalisme comme " the processes by which immigrants forge and sustain multi-stranded social relations that link together their societies of origin and settlement ". Nous considérons donc le transnationalisme comme la construction d'un espace- 
temps migratoire dans lequel des liens plus ou moins forts sont entretenus entre les membres (Glick Schiller et al., 1992 ; Portes, 1997), et dans le cas des familles roms, d'un réseau de parenté identifié et en continuelle redéfinition. Ces " espèces d'espace " (Pérec, 2000) que l'on peut qualifier de "labiles " font écho aux réflexions de Tarrius (1992) à propos des "territoires circulatoires " qui se remodèlent en fonction des événements, des temporalités migratoires.

\section{Les femmes roms : nouvelles actrices de la migration}

Une des caractéristiques de l'évolution des mouvements migratoires des Roms est la féminisation des flux. Les femmes rom qui migrent constituent aujourd'hui une part importante de la population des Roms roumains présents en France. D'une migration économique à forte dominante masculine, les migrations des Roms roumains se sont peu à peu transformées en migration familiale où, le plus souvent, la femme, accompagnée des enfants du foyer, rejoint son conjoint. Ainsi, en région parisienne, $50 \%$ des Roms habitant en bidonville ${ }^{8}$ et squat sont des femmes et des enfants (Romeurope, 2012). Cette évolution est particulièrement visible au sein des structures associatives où les femmes s'investissent de manière croissante. Dans ses travaux, De Gourcy (2014) a montré que pour les femmes, la migration est une solution pour améliorer les conditions de vie; dans le cas des femmes roms, la migration est aussi un moyen de s'émanciper de contraintes liées à la hiérarchie sociale et aux traditions familiales.

Le phénomène de féminisation des flux migratoires se manifeste dans la sphère associative où le public " actif " des collectifs se féminise également de façon considérable. Nous verrons que ces femmes ont un rôle majeur dans l'organisation et le déroulement de l'événement festif, aussi bien dans la tradition des familles que dans l'organisation de manifestations qui touchent un plus large public, opérant ainsi une externalisation des pratiques culturelles de la sphère privée vers la sphère publique et à une mise en visibilité des pratiques. D'une manière générale, les recherches portant sur la féminisation des flux migratoires (Veith, 2005 ; Amoranitis, Gatugu et Lanço, 2004) ont démontré I'investissement actuel et la grande visibilité des femmes migrantes dans les activités culturelles associatives dans les pays d'installation où elles tentent de concilier une volonté de conserver des liens forts avec leur communauté avec des projets de vie individuels d' " ascension sociale " (Veith, 2005 : 53). Cette mise en tension des traditions et des aspirations de chacune d'entre elles contribuent très largement à la remise en question des fonctions qui leur sont attribuées au sein de la famille. Martiniello (1995) fait le même constat à propos de la participation et de la représentation féminine dans les associations de migrants en Belgique, auparavant caractérisées par une forte présence masculine. Comme pour d'autres femmes migrantes d'origines diverses, les associations, notamment dans les pays d'installation, constituent de plus en plus un lieu ressource, de développement de compétences et de promotion ${ }^{9}$.

Bien que cet investissement associatif n'aboutisse pas encore à un processus d'individuation poussé pour les femmes roms, il montre dans tous les cas l'évo-

8 Appelés platz par les Roms roumains.

9 Source : GRDR. 
lution de leur place au sein de la structure sociale et familiale. La migration peut être alors considérée comme un révélateur des transformations des modèles familiaux traditionnels où la femme joue un rôle de plus en plus fondamental dans les processus d'insertion locale et dans le maintien de l'unité familiale. Par leurs différents investissements, ces femmes roms migrantes participent, de fait, à la légitimation de la présence familiale et révèlent des ancrages locaux, très souvent méconnus. Les observations réalisées sur le terrain ont montré que ces femmes prennent véritablement à cœur, un rôle nouveau de " médiatrice " entre la communauté et les autorités locales.

\section{Panorama historique de l'associationnisme rom à l'échelle française et de la région parisienne}

L'associationnisme rom est différent de celui d'autres populations migrantes qui construisent en amont leurs propres réseaux associatifs, comme c'est le cas notamment pour les immigrés portugais (Cordeiro et Hily, 2000 : 59-76) ou encore marocains (Dumont, 2007). Dans le cas des Roms, il s'agit d'un mouvement en grande partie exogène aux populations et la création d'associations ou encore de collectifs locaux est souvent le fruit de personnes non rom. En revanche, I'implication des Roms, une fois la structure créée, laisse entrevoir un investissement particulier et une participation mesurée aux négociations collectives. Les associations roms voient leurs modes d'organisation et leurs revendications sociales et politiques évoluer au fil du temps. Aussi, le paysage de l'associationnisme rom en France se remodèle en fonction de l'intensité des mouvements migratoires qui caractérisent certaines périodes et des politiques migratoires françaises qui tentent de contrôler les flux et les installations.

\section{Associationnisme rom en France}

Les premières associations exogènes voient le jour dans les années $1960^{10}$ avec un essor remarquable dans les années 1970. Cette période, que I'on peut qualifier de " mode associationniste " (Liégeois, 2007 : 203) est caractérisée non seulement par une augmentation significative des structures associatives qui s'occupent des Roms, mais aussi par une transformation du type de prise en charge. En effet, les principaux acteurs recrutés au sein de ces structures, auparavant bénévoles dans leur grande majorité, sont alors des médiateurs sociaux et des professionnels du social. La prise en charge des Roms devient une réelle " tutelle associative " passant d'un humanisme paternaliste à un " humanisme technocratique" (Liégeois, 2009 : 68). Ces premières structures, qui ont leur berceau à Paris, se généralisent petit à petit à l'ensemble du territoire français. Au-delà de la défense et de la promotion culturelle, elles deviennent un acteurclé dans les relations entre l'État et les Roms et tentent d'équilibrer leurs actions entre ces deux instances.

10 Ce mouvement s'établit aussi dans la majeure partie des pays d'Europe occidentale à la même période, illustrant ainsi une montée significative de la " problématique rom " sur le continent européen. Les premières préoccupations de ces structures étaient de natures sociales et éducatives. Le spectre de leurs activités s'élargit très rapidement à d'autres thématiques comme celle de la santé, des aides sociales, des activités professionnelles, etc. 
Les associations créées par les Roms font également leur apparition à partir des années 1960. Ainsi, en 1962 est créée "I'Association des Gitans et des Tziganes de France ", en 1968 "l'Association nationale desTsiganes de France ", I' " Office national des affaires tsiganes " et le "Bureau tsigane " en 1973. Les décennies 1980 et 1990 voient aussi le nombre d'associations portées par des Roms augmenter avec la création de la "Fédération tsigane de France " en 1981 ou encore le "Mouvement confédéral tsigane " en 1992. Ces associations sont à l'origine de nombreuses créations d'autres structures soutenues par des non-Roms, illustrant le fort poids du travail communautaire entre population cible et acteurs sociaux. Pendant près de vingt ans, les associations endogènes vont timidement augmenter, la plus significative d'entre elles étant " la Voix des Rroms " créée au printemps 2005 et dont le siège est à Paris. Son slogan "Les Rroms debout et acteurs " montre son enjeu principal et insiste sur sa particularité dans la masse d'associations existantes aujourd'hui, à savoir être portée par des personnes appartenant à la communauté et ne pas être " dépendante " d'autres acteurs extérieurs à la population plus globale des "Rroms ". Promotion culturelle et autonomie politique (Asséo, 2004 : 76) sont donc les missions premières du collectif qui se dit appartenir à I'“ intelligentsia romani ».

L'associationnisme rom en France prend donc un double visage : les structures créées " pour les Roms " et celles conçues " par les Roms ". Ces processus, endogènes et exogènes, entretiennent et renouvellent le tissu associatif et ce, à plusieurs échelles (du national au local, mais aussi à l'international). L'évolution des structures associatives, depuis les années 1960, montre que d'une volonté de créer des lieux "pour soi " et d'entre-soi, les associations exogènes ont pris le relai et se sont transformées, peu à peu, en lieu de visibilité culturelle et d'action politique (Cossée, Lada et Rigoni, 2004) par procuration.

\section{Les associations roms en région parisienne}

En région parisienne, il n'existe pas de structures associatives qui s'occupent uniquement des Roms roumains. Le critère de la nationalité y est moins important que celui de l'appartenance ethnique à la communauté rom. En revanche, nous savons d'une part, que les Roms roumains, de par leur nombre, sont majoritaires sur les autres nationalités; et d'autre part, que le travail des médiateurs sociaux doit intégrer cette variable, notamment dans la constitution des dossiers de régularisation ${ }^{11}$. C'est pourquoi, grâce au dialogue qui s'instaure entre les médiateurs et les Roms, la nationalité des migrants peut être facilement connue, ce qui permet de se focaliser sur les Roms de Roumanie.

\footnotetext{
11 Précisons que si la nationalité est exigée dans toute procédure de régularisation, I'appartenance ethnique est proscrite. C'est pourquoi, il est très difficile de savoir avec exactitude le nombre de Roms roumains présents en France qui font une demande de régularisation de situation. Seul le nombre de Roumains faisant une demande peut être connu. À cela s'ajoutent les Roms qui n'engagent pas de démarches administratives et qui ne sont pas connus des structures de prise en charge associatives.
} 
Les structures associatives en région parisienne ${ }^{12}$ décrivent un jeu d'échelle. Chacune d'entre elles intervient dans des domaines distincts et possède une visibilité plus ou moins grande sur la scène politique et sociale. Cette visibilité est directement liée à l'insertion des structures dans des réseaux de plus grandes ampleurs, notamment à l'échelle européenne. C'est le cas de deux associations qui chapeautent la majeure partie des structures et des collectifs de la région parisienne et de l'ensemble du territoire français. La FNASAT (Fédération nationale des associations solidaires d'action avec les Tsiganes et les Gens du voyage) et Romeurope sont en charge d'harmoniser les actions et de représenter les structures - plus petites - auprès d'institutions (souvent politiques) nationales et européennes. Situées dans le 19e arrondissement à Paris, elles sont un lieu clé pour un public large (associatifs, bénévoles et Roms) et elles ont une place majeure sur l'échiquier politique (Kepel, 1991 ; Kastoryano, 1988) à différentes échelles.

Bien que les collectifs locaux aient une autonomie dans leurs activités, les deux instances fédératrices veillent à ce que les manifestations culturelles soient organisées à la demande des Roms. Elles leur garantissent un rôle d'acteurs de premier plan, aussi bien en amont qu'en aval des rassemblements festifs, et travaillent continuellement au changement du regard porté sur ces populations, souvent considérées comme des "passifs " dans l'opinion publique. L'enjeu est de "faire lien " entre des populations vivant sur un même territoire, de créer une " empathie interculturelle " (Montandon, 2001) et d'éviter une fabrique identitaire extérieure. Les Roms sont considérés, par ces associations et collectifs, comme des protagonistes de moments festifs pour présenter leurs traditions et leur culture.

\section{La dimension festive et culturelle comme mode de contestation politique et de visibilité sociale}

La fête (Paćiv en romani) est avant tout un moment de partage avec autrui. Les moments festifs, dans leur sens global, soulignent l'entraide et l'hospitalité. Chez les Roms, cette solidarité n'est que la continuité d'un système basé sur l'échange et la redistribution, comme en témoigne le partage des bénéfices issus des transactions commerciales, qui se fait principalement sous deux formes entre les membres de la fratrie, à savoir la répartition financière et/ou l'organisation de réunions familiales (Williams, 1985 : 129). Seulement, la visibilité de ces pratiques et manifestations culturelles a elle aussi évolué - notamment sous les effets conjugués de la migration et des actions associatives - passant ainsi de la sphère privée et familiale à la sphère publique (Cordeiro et Hily, 2000 : 60) depuis les années 1990.

12 Parmi toutes les associations qui sont membres de la FNASAT et de Romeurope, seules celles qui travaillent spécifiquement auprès des Roms migrants en région parisienne ont été sélectionnées. En effet, certaines d'entre elles ne s'occupent que des Gens du voyage français et ont d'autres problématiques que celles qui nous intéressent, même si du point de vue de la participation associative ces structures et ce public constituent un matériau très intéressant. Sur la totalité des départements composant la région parisienne, quinze associations et collectifs locaux ont été recensés. Seules la FNASAT et Romeurope, instances fédératrices, sont localisées dans Paris intra-muros. Les autres structures, collectifs et relais se répartissent de façon assez équilibrée sur le territoire régional montrant une prise en charge harmonisée au sein du territoire parisien. 
Ces dimensions culturelles ont aujourd'hui une place importante dans le champ politique et associatif et participent des actions de reconnaissances, même si elles engagent moins la rencontre "physique ". La culture rom a la particularité d'être orale. Depuis les années 1990, et plus particulièrement dans les années 2000 (Liégeois, 2007 : 91), les types de support pour la diffusion s'élargissent afin de toucher un public plus large et populaire. Cette diversification des modes de transmission facilite la diffusion de l'expression identitaire. L'usage des médias électroniques inscrit la culture rom et sa dimension festive dans un contexte mondialisé et ces nouveaux outils contribuent maintenant à " des logiques de mise en représentation du soi collectif et de la mobilisation identitaire " (Rigoni, $2010:$ 7). Cette mise en visibilité de soi dans l'espace public, corrélative de l'émergence d'un mouvement tsigane à partir des années 1970 (Cossé, 2010 : 57), utilise donc différents supports, dont celui de l'internet, pour porter une parole collective (ibid.). Les travaux de Cossé (2010) sur les médias roms permettent de façon très nette de voir l'essor considérable de l'usage d'Internet et de la revendication par le web, au détriment de médias plus traditionnels tels que les journaux. De nombreux blogs, comme celui du Virtual Roma Network - créés à l'initiative de population rom -, voient le jour (une dizaine en France pour l'année 2007 par exemple) et œuvrent à la visibilité des actions roms transnationale.

\section{Des événements de mise en scène identitaire}

Les manifestations culturelles qui se déroulent en région parisienne pour engager la rencontre et l'échange culturel entre la population locale et les Roms mobilisent différents types de ressources. Toutes participent de l'inscription spatiale et sociale des Roms, même en contexte de migration et de présence éventuellement non durable. Deux types de manifestations ont retenu notre attention : celles qui s'inscrivent dans un mouvement international et dont la planification est réitérée chaque année à la même période ; et celles organisées localement, ponctuellement dont la visibilité est moindre. Avec ces exemples, nous verrons la nature et le degré de l'investissement des familles roms dans ces différentes manifestations ainsi que l'impact qu'elles ont sur leur quotidien.

\section{De la visibilité locale à la représentation internationale}

La première Roma Pride a eu lieu en octobre 2011 à Paris et se reproduit chaque année à la même période. C'est une manifestation basée à Paris, mais dont la mobilisation est nationale et internationale : elle se déroule simultanément dans les capitales de quinze pays européens, dont le Danemark, la Turquie, le Portugal, la Pologne, la Roumanie, la Bulgarie, I'Ukraine, etc. À Paris, elle est en général orchestrée par I'Union Française des Associations Tsiganes, SOS Racisme, I'Union des Étudiants Juifs de France (UEJF) et la Confédération étudiante. En plus d'être un moment de visibilité publique relayé par les médias, la Roma Pride encourage fortement la revendication politique et sensibilise le public à différents problèmes que rencontrent aujourd'hui la population rom migrante et plus généralement les Tsiganes en France, montrant ainsi que les intérêts collectifs sont prioritaires sur les enjeux particuliers à chaque groupe lors de ce type de manifestation de grande ampleur. Celle-ci porte un message clair avec des objectifs précis, et pour transmettre ces revendications auprès 
d'un public large, les ressources culturelles sont largement mobilisées. Ainsi, des grandes figures de l'art et de la musique rom et tsigane - symboles culturels reconnus internationalement - sont invitées pour porter la voie rom. Signe du caractère transnational de l'événement, ces personnalités appartiennent toutes à la population rom, mais sont de nationalité différente ${ }^{13}$ et ont été sollicitées pour leur notoriété et pour rassembler un public hétéroclite.

La dénonciation des actes racistes et des discriminations raciales envers les Roms constituent le premier point de revendication. La participation de SOS Racisme au rassemblement va d'ailleurs en ce sens. Ce motif de contestation est, pour sa part, international puisqu'il est repris dans l'ensemble des récriminations de tous les collectifs de chaque pays où se déroule la Roma Pride. Cette solidarité qui dépasse les frontières et qui œuvre pour une cause commune s'élève contre la "persécution transnationale " dont les Roms font l'objet (Bergeon, 2010).

Cette persécution se traduit, entre autres sur la scène européenne, par des possibilités de déplacements réduites pour les ressortissants roumains. La deuxième revendication de la Roma Pride de 2012 dépasse donc la " revendication particulariste " et pointe plus globalement du doigt la place accordée à la Roumanie dans le contexte européen du point de vue de la libre circulation des personnes. Rappelons que, pour la France, la Roumanie était encore dans sa période transitoire jusqu'au 1 ${ }^{\text {er }}$ janvier 2014 concernant les déplacements de ses ressortissants et que ces derniers étaient dans l'obligation de posséder une autorisation de travail pour exercer en dehors des frontières de leur pays ${ }^{14}$. La question de la circulation des Roms a été un des points à l'origine de la mesure transitoire. Les craintes d'un envahissement et d'une mobilité spatiale non contrôlée ont poussé les instances de I'Union européenne à ériger des conditions d'entrée spécifiques et non communes pour l'ensemble des citoyens de Roumanie. Au-delà de la circulation des Roms et de leur présence dans les principales capitales de l'Europe de l'Ouest, c'est bien la place qui est accordée à la Roumanie dans I'Union européenne qui est largement critiquée.

Les autres revendications concernent spécifiquement le cas français et dénoncent les démantèlements de camps sans solution de relogement. Depuis 2010, ces opérations entreprises par le gouvernement français se sont multipliées. En 2012, 9169 Roms ont été délogés de leurs lieux de vie ${ }^{15}$. Plus globalement, ces protestations défendent la révision de la stratégie nationale

13 Citons entre autres, le réalisateur français Tony Gatlif, le groupe de musiciens Taraf de Haidouks de Roumanie, la Fanfare Vagbontu de Moldavie, et Titi Robin, musicien français influencé par la musique tsigane.

14 Dans les textes réglementaires, tout citoyen européen peut traverser et vivre dans un autre État européen que le sien sans demander d'autorisation de séjour. Cependant, la France est un cas spécifique dans la mesure où au-delà de trois mois, celle-ci impose aux autres citoyens européens présents sur son territoire des justifications de ressources financières stables et, pour les ressortissants roumains et bulgares, elle impose l'obtention d'un permis de séjour pour pouvoir travailler.

15 Source : Association Européenne pour la Défense des Droits de l'Homme. Rapports consultables à l'adresse suivante : http://www.aedh.eu/Recensement-des-evacuations.html 
d'insertion $^{16}$ des Roms en France qui ne propose pas de dispositions ciblées et qui insiste, au contraire, sur l'accès au dispositif de droit commun. En France, ce plan prévoit, entre autres, un accès facilité au système de soin, à l'éducation, à I'emploi et au logement ${ }^{17}$ dans le cadre du droit commun.

Les fêtes locales constituent un autre type de manifestations culturelles et privilégient la proximité. Si elles ont aussi pour objectif la promotion culturelle et la rencontre, elles s'occupent néanmoins de problématiques locales spécifiques à chaque lieu d'installation des familles roms. Pour présenter ces fêtes, l'exemple du collectif "Les Allumeurs " est significatif. Une des dernières manifestations culturelles date de février 2013, celle-ci était intitulée "Dukreben " (" bonne aventure " en langue romani) et son slogan d'accroche était "Venez rêver, échanger, rencontrer, danser, goûter, à l'occasion d'un après-midi festif avec nos amis roms ". Elle a rassemblé autour d'un public varié, Roms et chercheurs. L'implication des Roms dans cette journée était une priorité pour le collectif "Les Allumeurs " et ils ont d'ailleurs animé le repas, les danses coutumières ; ils étaient présents tout au long de la journée. Comme dans le cadre de la Roma Pride, les ressources culturelles ont été la charnière de cette journée.

D'autres fêtes locales engagent de façon plus importante les Roms migrants et la population locale et ont pour objectif de pacifier les relations de voisinage, notamment lorsqu'il y a des installations irrégulières comme c'est le cas avec les lieux d'habitat précaire de type "bidonville ". Ces rencontres tentent de favoriser la co-présence, et d'aller au-delà d'une juxtaposition des individus les uns à côté des autres, vers un "vivre ensemble " (Lévy et Lussault, 2003). Ces fêtes touchent donc un public restreint, celui qui est confronté à une présence qui souvent dérange et interroge, à cause de son caractère inconnu.

Les départements limitrophes à l'intérieur de la région parisienne sont concernés par le développement de ces lieux d'habitat précaire. C'est le cas, par exemple, de Ris-Orangis dans I'Essonne qui a accueilli un de ces lieux de vie. L'ASEFRR (Association Solidarité Essonne Familles Roumaines et Rroms), aidée de I'association PEROU (Pôle d'Exploitation des Ressources Urbaines), a

16 Ces stratégies d'insertion ont été imposées par I'Union européenne. Chaque pays membre doit proposer un plan d'insertion et dresser des bilans qui sont soumis aux instances européennes.

17 L'exemple le plus significatif des opérations de relogement est sans doute celui des " villages d'insertion " dans le cadre des Maîtrises d'œuvre urbaine et sociale (MOUS). Ce dispositif en test dans le département de la Seine-Saint-Denis en région parisienne a été élaboré en parallèle au démantèlement de trois lieux d'habitat précaire à SaintOuen, Aubervilliers et Saint-Denis. Une sélection d'environ vingt familles par site s'est opérée, et celles-ci ont intégré ces villages d'insertion, situés dans la même commune que leur lieu d'installation précédente. Ces villages sont surveillés continuellement et les visites sont interdites sauf sur dérogation du gestionnaire. Certains auteurs comparent ces lieux de résidence à de réelles cités de transit (Pétonnet, 1985). Ladite insertion se fait en trois étapes : la première, appelée "Pré MOUS ", est un temps d'accueil des familles qui s'installent en caravane dans le village durant la mise en place d'un habitat sédentaire modulaire. La deuxième étape, "Mous d'insertion ", est dédiée à l'aménagement des habitations modulaires. En parallèle, les activités de "sensibilisation " se mettent en place : I'insertion professionnelle et l'individualisation des comportements sont la priorité (apprentissage de la gestion d'un budget, etc.) (Legros, 2010). Enfin,

lors de la troisième étape, l'État et les collectivités locales qui sont en charge de chacun des villages, délivrent des titres de séjour et offrent un logement de droit commun aux familles qui ont su remplir l'ensemble de ces conditions d'insertion, à la seule condition que le chef de famille ait signé un contrat de travail. 
organisé fin 2012 l'inauguration de "l'Ambassade ", bâtiment en bois pouvant servir de salle collective ou d'accueil à l'ensemble des Rissois. Cette fête a rassemblé les Roms habitant le lieu, la population locale et les membres des associations; des spectacles de musiques roms y ont été organisés. Les festivités ont rassemblé Français et Roms, ces derniers appréciant particulièrement que leurs voisins viennent sur leur lieu de vie. Cette initiative de l'ASEFRR et du PEROU, puis la mobilisation de la population locale suite à ces festivités, ont permis aux habitants de ce "bidonville " de rester sur le lieu de vie puisque l'expulsion prévue en décembre 2012 a été annulée par les autorités ${ }^{18}$.

Ces manifestations ne sont que des exemples parmi de nombreuses rencontres planifiées en région parisienne. Nous avons vu, à travers ces trois cas, que les festivités peuvent s'organiser à plusieurs échelles : celle de la ville avec une diffusion vers l'international, à l'échelle du quartier ou au niveau du lieu d'habitation. Elles rassemblent des publics différents de par la médiatisation qu'elles engendrent et à l'image de leur ampleur, elles sensibilisent à des problématiques locales ou bien à des enjeux transnationaux.

\section{Les bénéfices des manifestations culturelles pour les Roms}

L'hétérogénéité de ces rencontres festives, tant d'un point de vue du contenu que de leur ampleur, offre un terrain pertinent pour discuter le lien entre visibilité et impact pour les Roms à l'échelle locale. On pourrait aisément supposer que plus la manifestation est médiatisée, plus la portée du message et ses retombées seraient positives pour les Roms. Or, le discours des acteurs associatifs est plus modéré. En effet, ils pointent le caractère sensationnel des événements de grande ampleur où la curiosité du public prévaudrait sur l'investissement politique et la défense d'une cause. Cependant, nous avons vu que ces moments de rencontre participaient pleinement de la politisation des ressources culturelles et que celles-ci étaient considérées comme un outil de sensibilisation et d'engagement politique. Ce type de manifestations prône alors la "romitude " (Plésiat, 2010 : 124) au point parfois d'instrumentaliser des pratiques culturelles et d'en faire un outil médiatique. Ce sont des événements largement " publicisés " (Amicelle et Bigo, 2010 : 102) qui visent plutôt la découverte culturelle que la protection ou une éventuelle position de contestation politique, du moins pour les participants qui ne sont pas d'origine rom.

En revanche, les acteurs associatifs accordent une place de choix aux festivités qui se déroulent sur le lieu de vie des familles roms, même si celui-ci est souvent temporaire et susceptible d'être détruit. En lien direct avec le voisinage, la problématique touche sensiblement la population locale. La proximité géographique et sociale des familles fait que celles-ci ne peuvent passer inaperçues dans la commune ou le quartier. Le lien existe donc même s'il n'est que visuel. Dans tous les cas, la présence des Roms à l'échelle locale instaure, malgré tout,

18 Ce lieu d'habitat précaire a été détruit le 3 avril 2013. Des familles ont été relogées alors que d'autres sont parties vers d'autres lieux en région parisienne. La commune de Ris-Orangis a cependant octroyé un " terrain de stabilisation " aux familles qui souhaitaient rester à proximité de leur ancien lieu de vie. L'association PEROU, dans un communiqué, a précisé qu'à l'instar de l'ancien " bidonville ", elle " amènerait la ville " jusqu'à ce nouveau terrain, isolé et dépourvu de tout équipement, c'est-à-dire qu'elle fera son possible, par de menus aménagements, pour rendre vivable et humaniser ce lieu. 
de la cohabitation, que celle-ci dérange, interroge ou sensibilise. Cet impact plus prégnant des manifestations locales est aussi mis en avant par les Roms qui non seulement valorisent leur participation à I'organisation de ces moments festifs, mais aussi insistent sur le caractère " intime " que leur permettent ces moments de rencontre sur leur lieu de vie. Ils soulignent l'intérêt des manifestations de grande ampleur, mais les différencient largement de celles, plus locales, où la proximité sociale devient un outil et une ressource. Le caractère "impersonnel " des grandes festivités les empêcherait, en quelque sorte, de nouer réellement du lien social avec la société d'accueil. Ainsi, selon les acteurs associatifs parisiens, l'impact pour la population rom à l'échelle locale est bien moindre dans le cas des grandes manifestations et "l'après-manifestation " n'engage pas autant de sensibilisation et d'engagement qu'un événement festif local.

Ces observations interrogent de fait l'importance et les impacts des sociabilités externes et internes (Authier, Bensoussan et Grafmeyer, 2001 ; Lévy et Lévy-Vroelant, 2001 ; Grafmeyer, 1998 ; Cortés Alcalá, 1995) comme les " rapports à autrui " et les " échanges avec la société d'installation ", tant dans la sphère domestique que publique. La relocalisation des événements festifs sur le lieu de vie entraîne un double processus. Dans un premier temps, celui d'une construction d'un chez soi pour les familles malgré leurs conditions de vie et de logements insalubres. Recevoir, organiser une fête dans le squat ou le " bidonville " permet d'engager I'hospitalité et l'accueil, élément fondamental des relations humaines et de l'acte d'habiter un lieu. Rappelons que le droit à I'habiter, dans le cas des Roms, est très largement remis en cause par la société et que le devoir d'accueil est souvent refoulé. Dans un second temps, ces festivités planifiées sur le lieu d'habitation permettent aux riverains de vivre l'intimité des familles. Ces sociabilités internes donnent lieu à une sensibilisation directe à la précarité résidentielle et économique qui touche les Roms. Le caractère festif et la mise en valeur des pratiques quotidiennes lors de ces rencontres de proximité permettent aussi de dépasser cette vision pauvre et/ou dangereuse du squat ou du " bidonville ". Une femme du bidonville disait, en fin de journée sa grande surprise d'avoir vu tant de monde, sa joie de cette fête, le plaisir de ses enfants, de ces rencontres avec ces "Français " venus chez elle, dans sa cabane. Elle a pu montrer ses efforts pour que tout soit propre. Ils en ont été surpris, et le lui ont dit. Une autre femme disait son immense bonheur, " jamais je n'avais imaginé ", car l'institutrice de l'un de ses enfants est venue chez elle prendre le thé, parler de la scolarité ${ }^{19}$.

Les discours des Roms et des acteurs associatifs laissent donc transparaître des retombées différentes en fonction de la visibilité des festivités. Paradoxalement, moins la visibilité est forte, plus les conséquences sont perceptibles et touchent de façon directe les Roms. Néanmoins, même si ces festivités génèrent des engagements variables de la part des participants et du public, elles ont le mérite de réunir des populations qui souvent s'ignorent ou sont en situation de conflit au quotidien et qui font l'objet de fortes représentations dépréciatives. Les ressources culturelles sont alors un tremplin pour fédérer les personnes. La proximité, l'éloignement ou la délocalisation des événements festifs ont des impacts certains sur la sensibilisation du public, comme le précise la coordinatrice principale de Romeurope à Paris :

19 Source : ASEFRR. 
"Sur le terrain ou en dehors du terrain, ce n'est pas pareil. Le lieu d'organisation de la manifestation a un rôle très important. On ne sensibilise pas de la même manière si on organise sur le terrain ou pas. L'impact sur les représentations du voisinage est direct et cela joue sur l'hospitalité de manière immédiate " (entretien réalisé à Paris en 2013).

Ces événements festifs et culturels véhiculent des messages dont les problématiques diffèrent selon l'intensité de la manifestation et apportent aux familles des retombées le plus souvent éphémères. Ils s'insèrent aujourd'hui très peu dans le champ politique dans la mesure où les enjeux et les effets de la médiatisation sont de moins en moins efficaces.

Du côté des Roms, ces moments de rencontre leur permettent d'aller au-delà d'une seule " sociabilité communautaire " trop prononcée qui les rassemblerait entre eux et ce, dans des lieux confinés, isolés géographiquement et socialement. Cette dialectique de l'ouverture et de la fermeture aux autres montre inévitablement que la migration s'insère dans un processus continu et non scindé en différentes étapes indépendantes les unes des autres. En effet, si l'entre-soi alimente la cohésion du groupe et les attaches à la Roumanie, les relations développées avec la population locale sont autant de ressources dans la conduite du projet migratoire. La présentation de soi, par le biais des ressources culturelles, fait non seulement partie d'une stratégie d'affirmation identitaire, mais aussi d'une référence continuelle au village et aux membres de la communauté restés en Roumanie.

\section{L'engagement associatif à la lumière du temps de la migration et des situations résidentielles}

Nous avons vu que I'histoire de l'associationnisme rom présentait une construction fortement exogène. Au vu des entretiens qui ont été effectués avec les Roms et les médiateurs sociaux, il apparaît que trois facteurs conditionnent particulièrement l'implication des Roms dans les associations et dans I'organisation des événements festifs.

Premièrement, la durée de présence et l'arrivée récente ou non à Paris influencent fortement l'engagement associatif. Même si les réseaux migratoires sont bien structurés tout au long du processus migratoire, les Roms nouvellement arrivés dans la capitale mettent du temps à prendre leurs marques et à découvrir un environnement souvent méconnu. L'appel aux structures associatives se fait, dans un premier temps, pour des raisons liées à l'installation et aux différents recours possibles pour faire durer la présence en France. Ainsi, même si les associations jouent très vite un rôle moteur dans le quotidien des Roms roumains nouvellement arrivés, ce sont bien les solidarités communautaires et familiales qui prévalent sur un engagement associatif. Dans ces cas-là, les structures font office de ressources sociales, parfois économiques, pour ces nouveaux migrants afin qu'ils puissent s'installer et inscrire leur présence dans la ville. La participation associative nécessite donc un temps d'adaptation et c'est souvent l'impulsion d'autres Roms roumains vivant sur le même lieu d'installation ou faisant partie des réseaux communautaires en région parisienne qui influence, par la suite, l'engagement associatif et la participation aux manifestations culturelles. Ainsi, dans les premiers temps de la migration, les sociabilités 
internes et l'entre-soi sont plus forts que les activités extérieures qui ne sont pas en relation avec la migration et l'installation à Paris.

À l'opposé, les Roms qui sont présents en région parisienne depuis plusieurs mois, pour certains depuis plusieurs années, voient leur engagement grandir au fur et à mesure que leur présence se prolonge. Pour nombre de familles, les structures deviennent un point d'appui essentiel et un outil d'expression identitaire :

" L'impact temporel est très important. Les collectifs et les comités travaillent beaucoup plus avec les Roms installés depuis longtemps dans le cadre de la promotion culturelle, même s'ils sont dans l'irrégularité vis-à-vis de leur présence. Le moment festif n'est pas le moment de l'expulsion. La fête peut arrêter l'expulsion. Les Roms qui sont là depuis longtemps participent plus et mieux parce qu'ils arrivent à se projeter dans l'avenir grâce à ces manifestations culturelles et rencontres entre populations. C'est tout un chemin " (coordinatrice principale de Romeurope, entretien réalisé à Paris en 2013).

La durée de présence influence donc d'une part l'investissement des personnes dans les événements de rassemblement et d'autre part elle conditionne le ressenti lors de ces moments. L'ambiance et l'implication de la population locale participent au développement du sentiment d'appartenance locale et au statut d'habitant :

"Quand tu arrives à Paris, tu es étranger... et tu es Rom... C'est très difficile. Au début, c'est très difficile... après aussi, mais ce n'est pas pareil. On se retrouve et on essaie de vivre. On est ensemble. Ici c'est toujours mieux qu'en Roumanie. On n'a rien. C'est bien de rentrer, mais on n'a rien. Ici [à Paris], tu peux t'en sortir seul, mais c'est du courage et surtout tu ne sais pas comment ça va se passer. On habite tous là. On

a essayé de faire ça bien, oui comme on pouvait. On a des visites, des Français, ils travaillent à l'association pour nous. Ce n'est pas arrivé souvent, mais des Français sont venus nous voir, pas ceux de l'association, enfin si, ils étaient là, mais il y avait d'autres gens. C'était bien. Ils sont venus chez nous. C'est comme si tu reçois des voisins... mais bon, c'est ici... ce n'est pas le paradis. C'est bien que des gens s'intéressent à nous, on $n^{\prime} y$ croyait pas. Ils avaient même organisé un verre de l'amitié. C'est surtout nos femmes qui étaient contentes. Ça faisait longtemps qu'elles n'avaient pas reçu des gens chez elles et c'est important pour elle ${ }^{20}$ (entretien réalisé à Paris en 2012).

Le deuxième facteur influençant l'investissement et la participation associative est l'urgence liée aux situations d'expulsion. Le démantèlement des squats et des camps de Roms suivent une procédure stricte, quoique rendue peu visible par les pouvoirs politiques. Des réunions préparatoires entre forces de l'ordre, Préfet et élus locaux doivent être planifiées avant toute instruction. Les associations locales y sont souvent absentes bien que leur présence soit obligatoire. En effet, la circulaire du 26 août $2012^{21}$ stipule qu'en cas de décision de justice liée à une expulsion de camps, des rencontres préalables doivent être organisées en présence des acteurs associatifs locaux s'occupant des familles. Sauf évacuation

20 Les propos ont été réécrits de manière à ce qu'ils soient compréhensibles. Le prénom a été rendu anonyme.

21 Circulaire interministérielle NOR INTK1233053C du 26/08/2012 relative à I'anticipation et à l'accompagnement des opérations d'évacuation des campements illicites. 
immédiate, un délai peut être octroyé aux personnes vivant dans les camps, celui-ci pouvant aller jusqu'à plusieurs semaines.

Le caractère urgent influence de deux manières la participation associative. Dans un premier temps, l'avis d'évacuation peut engendrer des mouvements de revendication et de sensibilisation auprès de la population afin que celle-ci intervienne auprès des autorités locales. Ce fut notamment le cas pour le camp de Ris-Orangis déjà cité ci-dessus et plus récemment pour le " bidonville " du Samaritin situé à La Courneuve, démantelé en août 2015. Le plus vieux bidonville de France accueillait près de 300 personnes. Les revendications ont été multiples : publication d'une pétition par l'un des jeunes roms habitant le camp, médiatisation d'images et de paroles d'habitants du quartier, rassemblements, etc. Ainsi, sous la menace d'une expulsion, les fêtes et autres manifestations culturelles peuvent servir d'outil pour enrayer ou retarder la procédure administrative. La crainte du refoulement, qui est constante pour de nombreux Roms, les pousse alors à fortement s'impliquer et se ranger derrière les structures associatives, celles-ci leur servant d'appui et de " refuge " contre l'insécurité liée à l'expulsion.

En revanche, une autre conséquence peut être l'abandon ou le désinvestissement de ces lieux associatifs. En effet, l'illégalité de la présence, menacée par une procédure d'évacuation et un éventuel retour forcé au pays, instaure une grande crainte pour ces familles. La peur d'être retrouvées par les autorités les encourage souvent à fuir et à se désinvestir des manifestations culturelles visibles, préférant se fondre dans l'espace public. Ici, I'invisibilité de la présence leur confère l'opportunité de ne pas être localisées et d'échapper à la procédure d'expulsion.

Un dernier facteur intervient dans l'investissement associatif, c'est le type d'habitat. En effet, les médiateurs sociaux mentionnent à de nombreuses reprises l'effet des conditions de logement sur la participation que ce soit lors des événements festifs locaux ou à plus grande échelle. Les familles qui logent en squat et "bidonvilles " ainsi que dans les villages d'insertion s'inscrivent plus facilement dans une démarche associative et de promotion culturelle. À I'opposé, les Roms qui réussissent à accéder au logement de droit commun se détachent au fil du temps de ces structures. De nombreuses recherches ont mis en avant le lien étroit entre l'accès au logement et l'intégration des populations dans la société d'accueil (Gotman, 2004 ; Simon, 1998). Celles-ci montrent notamment qu'une situation stable au niveau de I'habitat et de l'emploi, concoure à se rendre invisible dans l'espace social :

"Par exemple, les Roms qui habitent maintenant en appartement sont les moins actifs vis-à-vis de la participation aux manifestations culturelles. Comme s'ils avaient dépassé un cap. Ils ne renient pas leurs appartenances, mais je pense que leur parcours est tellement difficile et rare que dans un premier temps ils soufflent. C'est une réussite pour eux et ils ne veulent surtout pas perdre ce qu'ils ont gagné. Mais c'est vrai que dans

l'ensemble, une fois les familles stabilisées, ce qui est de plus en plus rare, elles sont beaucoup moins actives culturellement. Je pense que ce n'est pas un manque de solidarité envers leurs compatriotes, c'est juste qu'ils se posent ". (coordinatrice principale de 
La diminution de l'investissement des familles roms pour lesquelles les situations professionnelles et résidentielles se sont stabilisées, ne correspond pas à un désengagement profond vis-à-vis de la situation plus globale des Roms ni à une mise en anonymat complète du migrant comme le suggère la tradition sociologique l'École de Chicago à propos de l'insertion des migrants dans la ville et de leur intégration à la société d'accueil (Grafmeyer et Joseph, 2004 ; Missaoui et Tarrius, 2006). L'amélioration des conditions d'habitat et des ressources ne provoque pas l'abandon total des compatriotes qui sont touchés par les "galères migratoires ". L'effet "caméléon " identifié par Chicago ne s'établit pas tant lors d'un passage entre citadins problématiques et citadins intégrés que lors d'ajustements quotidiens des capacités à se jouer des codes de la société d'accueil et des traditions communautaires en référence aux travaux anthropologiques menés au sein de l'École de Manchester. Malgré une faible participation, elles ne se désintéressent pas des problèmes rencontrés par leurs concitoyens nouvellement arrivés ou installés de façon durable. Seulement, I'entraide et la solidarité se manifestent autrement. Si les situations " transitoires ", caractérisées par la précarité résidentielle et économique, font largement appel aux sociabilités externes et aux recours associatifs, I'amélioration des conditions de vie et surtout l'insertion dans la société d'accueil laisse plutôt place à des sociabilités internes et communautaires où les solidarités familiales et privées prennent le pas. Ainsi, de nombreuses familles qui, aujourd'hui, ont une situation stable viennent en aide à ceux qui sont plus vulnérables. Les réseaux migratoires se formalisent et deviennent alors identifiables. Leurs réalités et leur efficacité participent à l'orientation des flux migratoires vers des destinations privilégiées (Diminescu, 2003) dont la région parisienne fait partie. Cette structuration en réseaux, peu visible, mais cependant bien organisée, vient pallier des difficultés d'installation sur le long terme, transformant les objectifs migratoires : les déplacements des Roms roumains s'insèrent aujourd'hui dans une logique de circulation migratoire et de banalisation des retours au pays (Bergeon, 2010 : 204). Les ressources mobilisées avant le départ, pendant la migration et dans la société d'installation montrent que les réseaux migratoires existent et se remodèlent en fonction des " aléas " du projet migratoire.

" Je suis arrivée à Paris en 2011. Mon quartier c'est le 20e. La galère... tous les jours la galère. II faut manger, s'occuper des enfants et trouver un endroit pour dormir ou juste être en sécurité. On n'est rien. Pourtant c'est beau ici. Au fur et à mesure, les commerçants du quartier me connaissent, parfois ils me donnent de la nourriture. On est arrivés à plusieurs. Un jour, Sara [une autre femme du village] m'a demandé de venir avec elle. Je l'ai suivie. Je crois que c'était dans le 19e donc pas très loin. Elle m'a fait rentrer dans un immeuble. Tu sais jamais encore j'avais fait ça. On est monté. Elle a sonné et on est entré. Et là ça sentait la Roumanie. Je ne sais pas comment expliquer. Ils nous ont aidées, parfois je travaillais pour des gens, mais c'était rare. Le plus qu'ils ont fait c'est parler et ça, ça fait du bien. Et puis aussi de voir que des gens comme nous au début ont réussi. Mais là je crois que ce n'est pas encore mon tour " (entretien réalisé à Paris en 2012).

Bien qu'ils apparaissent peu structurés (Nacu, 2010 : 148), ils permettent aux Roms d'avoir, par exemple, une activité économique même si celle-ci est très souvent illégale. Dans le même temps, ces réseaux permettent l'entraide et le soutien moral, montrant ainsi toute l'importance des solidarités communautaires comme le souligne Massey (1993 : 396) dans sa définition du réseau : " I'ensemble des liens interpersonnels qui lient les migrants, les anciens 
migrants, et les non-migrants dans les zones d'origine et de destination à travers les liens de parenté, amitié et origine communautaire ".

\section{Conclusion}

L'installation des Roms roumains en région parisienne $n^{\prime}$ engendre pas que des conflits auxquels nous habituent les médias depuis plusieurs années. Les familles sont également présentes de manière plus ou moins visible sur la scène publique par le biais des manifestations culturelles et de leur engagement associatif. Ces participations collectives révèlent la force des solidarités communautaires, I'attachement au groupe et l'affirmation identitaire en situation de migration.

Le paysage associatif rom en Île-de-France est très hétérogène. De grandes structures comme de petits collectifs s'assurent de la protection des familles qui vivent le plus souvent dans des conditions de vie déplorables. L'associationnisme rom se questionne aujourd'hui à travers "l'élite rom " qui se présente comme les défenseurs premiers de la population rom et insiste sur l'unité du peuple rom à travers le monde.

Au-delà d'une simple présentation du contexte associatif en région parisienne, cet article a défendu la thèse selon laquelle les manifestations culturelles de grande ampleur - donc très visibles - ont peu de conséquences directes sur le quotidien des Roms. Ce postulat est renforcé par le discours des acteurs associatifs qui prônent, en premier lieu, les actions ciblées et localisées pour faire évoluer des situations de vie souvent très précaires et instables. Ici, l'impact spatial des événements culturels a toute son importance. La manifestation locale est vue comme plus fédératrice dans la mesure où elle s'adresse à un public de proximité géographique et donc concerné par la présence des Roms dans l'espace local. Ces événements de petite ampleur peuvent avoir des conséquences concrètes dans I'accueil des familles à l'échelle des quartiers ou encore des communes. Ainsi, ces rencontres favorisent l'hospitalité car elles s'ouvrent à la mixité sociale, à la rencontre interculturelle malgré des représentations dépréciatives très ancrées dans l'opinion publique. Cet imaginaire envers les Roms et envers les Tsiganes en général - qui perdure depuis maintenant plusieurs siècles, notamment du fait de leur mobilité, est alimenté de façon continue par des discours politiques et médiatiques axés sur la répression et l'insécurité. Les politiques $d^{\prime}$ accueil ${ }^{22}$ implicitement créées en direction des populations roms renforcent une suspicion déjà très vivace. Ces instants festifs permettent, dans une large mesure, de travailler sur ces représentations collectives sur le court terme. Le facteur temporel est un élément important pour comprendre l'investissement associatif. Ceci montre notamment que les structures associatives sont un appui essentiel et une ressource dans la migration.

22 À titre d'exemple, nous pouvons citer la circulaire d'août 2012 en France relative à I'anticipation et à l'accompagnement des opérations d'évacuation des campements illicites qui visent implicitement les familles roms migrantes. 


\section{Références bibliographiques}

Amicelle Anthony et Bigo Didier (2010) Biopolitique et gouvernement des populations : perspectives autres, Paris, L'Harmattan, $147 \mathrm{p}$.

Amoranitis Spyros, Gatayu Joseph et Lanço Altay (2004) La vie associative des migrants : quelles (re)connaissances? Réponses européennes et canadiennes, Paris, L'Harmattan, 280 p.

Asséo Henriette (2004) Les Gypsy Studies et le droit européen des minorités, Revue d'histoire moderne et contemporaine, 5 (51-4bis), pp. 71-86.

Authier Jean-Yves, Bensoussan Bernard et Grafmeyer Yves (2001) Du domicile à la ville : vivre en quartier ancien, Paris, Anthropos, 214 p.

Bash Linda, Glick Shiller Nina and Szanton Blanc Cristina (1994) Nation Unbound:Transnational Projects, Postcolonial Predicaments, and Derritorialized Nation-state, NewYork, Gordon and Breach, 356 p.

Bergeon Céline (2010) Les Roms roumains en France. Entre politiques migratoires et politiques de non-accueil, Revue d'Études Comparatives Est-Ouest, 41 (4), pp. 197-211.

Bergeon Céline et Hoyez Anne-Cécile (2015) Être migrant et vivre en squat : la santé à l'épreuve des trajectoires résidentielles précaires, L'espace politique, 26 (2), [en ligne]. URL : http://espacepolitique.revues.org/3517

Cordeiro Albano et Hily Marie-Antoinette (2000) La fête des Portugais : héritage et invention, Revue Européenne des Migrations Internationales, 16 (2), pp. 59-76.

Cortés Alcalá Luis (1995) La cuestión residencial: bases para una sociología del habitar, Madrid, Editorial Fundamentos, $412 \mathrm{p}$.

Cossée Claire (2010) Médias tsiganes en France et en Hongrie : re-présentation de soi dans l'espace public, Revue Européenne des Migrations Internationales, $26(1)$, pp. 57-80.

Cossée Claire, Lada Emmanuelle et Rigoni Isabelle (2004) Faire figure d'étranger: regards croisés sur la production de l'altérité, Paris, Armand Colin, 319 p.

Coquio Catherine et Poueyto Jean-Luc (2014) Roms, Tsiganes, Nomades. Un malentendu européen, Paris, Khartala, 676 p.

De Gourcy Constance (2014) Femmes et mères dans la migration : destins individuels et destin collectif, in Natalia Ribas-Mateos et Véronique Manry, Mobilités au féminin. La place des femmes dans le nouvel état du monde, Paris, Karthala, pp. 181-194.

Delépine Samuel (2007) Quartiers tsiganes : I'habitat et le logement des Roms de Roumanie en question, Paris, L'Harmattan, $167 \mathrm{p}$.

Diminescu Dana (2003) Visibles, mais peu nombreux... : les circulations migratoires roumaines, Paris, Édition de la Maison des Sciences de l'Homme, 339 p.

Diminescu Dana (2001) L'installation dans la mobilité : les savoir-faire migratoires des Roumains, Migrations sociétés, 74, pp. 107-116.

Dumont Antoine (2007) La marocanité associative en France. Militantisme et territorialité d'une appartenance exprimée à distance, Thèse en géographie, Université de Poitiers, $624 \mathrm{p}$. 
Fibbi Rosita et D'Amato Gianni (2008) Transnationalisme des migrants en Europe : une preuve par les faits, Revue Européenne des Migrations Internationales, $24(2)$, pp. 7-22.

Glick Schiller Nina, Basch Linda and Szanton Blanc Cristina (1992) Transnationalism: A New Analytic Framework for Understanding Migration, Annals of the New York Academy of Science, 645, pp. 1-24.

Grafmeyer Yves (1998) Logement, quartier, sociabilité, in Marion Segaud, Catherine Bonvalet et Jacques Brun Éds., Logement et habitat, l'état des savoirs, Paris, La Documentation Française, pp. 347-354.

Grafmeyer Yves et Joseph Isaac (2004) L'École de Chicago. Naissance de l'écologie urbaine, Paris, Flammarion, $377 \mathrm{p}$.

Gotman Anne (2004) Ville et hospitalité. Les municipalités et leurs étrangers, Paris, MSHS, 492 p.

Guest Milena et Nacu Alexandra (2008) Roms en Bulgarie, Roms en Roumanie quelle intégration ?, Méditerranée, 110, pp. 105-115.

Kastoryano Riva (1988) Paris-Berlin : Politiques d'immigration et modalités d'intégration des Turcs, in Rémy Leveau et Gilles Kepel, Les Musulmans dans la Société française, Paris, Presses de la Fondation Nationale des Sciences Politiques, pp. 141-169.

Kepel Gilles (1991) Les banlieues de I'Islam. Naissance d'une religion en France, Paris, Éditions du Seuil, $425 \mathrm{p}$.

Legros Olivier (2010) Les " villages d'insertion " : un tournant dans les politiques en direction des migrants roms en région parisienne ?, Asylon(s), 8, [en ligne] consulté le 01/07/2016. URL : http://www.reseau-terra.eu/article947.html

Legros Olivier et Rossetto Jean (2011) La " question rom " en Europe aujourd'hui : regards croisés et mises en perspective, Etudes tsiganes, 16 (2), pp. 4-25.

Lévy Jean-Pierre et Lévy-Vroelant Claire (2001) Habiter son logement : présences et usages, in Jean-Yves Authier Dir., Du domicile à la ville : vivre en quartier ancien, Paris, Anthropos, pp. 51-71.

Lévy Jacques et Lussault Michel (2003) Dictionnaire de la géographie et de l'espace des sociétés, Paris, Belin, 1033 p.

Liégeois Jean-Pierre (2009) Roms et Tsiganes, Paris, La Découverte, 125 p.

Liégeois Jean-Pierre (2007) Roms en Europe, Strasbourg, Éditions du Conseil de l'Europe, $311 \mathrm{p}$.

Martiniello Marco (1995) Le mouvement associatif des immigrés d'hier et d'aujourd'hui, Nouvelle tribune, 9, pp. 43-44.

Marušiakova Elena et Popov Veselin (2008) Les migrations des Roms balkaniques en Europe occidentale : mobilités passées et présentes, Balkanologie, 11 (1-2) ), [en ligne]. URL : https://balkanologie.revues.org/972

Massey Douglas S. (1993) Theories of International Migration: Review and Appraisal, Population and Development Review, 19 (3), pp. 431-466.

MIGREUROP (2012) Atlas des migrants en Europe : Géographie critique des politiques migratoires, Paris, Armand Colin, $144 \mathrm{p}$. 
Missaoui Lamia et Tarrius Alain (2006) Villes et migrants : du lieu-monde au lieupassage, Revue Européenne des Migrations Internationales, 22 (2), pp. 43-65.

Montandon Alain (2001) L'hospitalité : signes et rites, Clermont-Ferrand, Presses Universitaires Blaise Pascal, Centre de recherches sur les littératures modernes et contemporaines, $293 \mathrm{p}$.

Nacu Alexandra (2010) Les Roms migrants en région parisienne : les dispositifs d'une marginalisation, Revue Européenne des Migrations Internationales, 26 (1), pp. $141-160$.

Olivera Martin (2009) Les Roms migrants en France, Diversité, 159, pp. 179-188.

Pérec Georges (2000) Espèces d'espaces, Paris, Galilée, 185 p.

Pétonnet Colette (1985) On est tous dans le brouillard. Ethnologie des banlieues, Paris, Galilée, 329 p.

Plésiat Mathieu (2010) Les Tsiganes : I'intégration éprouvée, tome 2, Paris, L'Harmattan, $198 \mathrm{p}$.

Pons Emmanuelle (1995) Les Tsiganes en Roumanie : des citoyens à part entière?, Paris, L'Harmattan, $142 \mathrm{p}$.

Portes Alejandro (1997) Globalization from Below: The Rise of Transnational Communities, Princeton, Princeton University, $52 \mathrm{p}$.

Potot Swanie (2007) Vivre à I'Est, travailler à l'Ouest: les routes roumaines de I'Europe, Paris, L'Harmattan, $226 \mathrm{p}$.

Reyniers Alain (2008) La mobilité des Tsiganes en Europe : entre fantasmes et réalités, Hermès, 51 (2), pp. 107-111.

Reyniers Alain (1995) Quelques éléments pour une histoire des médias tsiganes, Études Tsiganes, 5, pp. 141-146.

Rigoni Isabelle (2010) Les médias des minorités ethniques. Représenter l'identité collective sur la scène publique, Revue Européenne des Migrations Internationales, 26 (1), pp. 7-16.

Robert Christophe (2007) Éternels étrangers de l'intérieur, Paris, Desclée de Brouwer, $452 \mathrm{p}$.

Romeurope (2012) "Les Roms migrants " en île de France. État des lieux provisoire des expériences d'hébergement et de logement d'habitants de squats et bidonvilles, Paris, Romeurope, $15 \mathrm{p}$.

Simon Patrick (1998) Le logement et l'intégration des immigrés, in Marion Segaud, Catherine Bonvalet et Jacques Brun, Logement et habitat : état des savoirs, Paris, La Découverte, pp. 327-335.

Tarrius Alain (1992) Les fourmis d'Europe : migrants riches, migrants pauvres et nouvelles villes internationales, Paris, L'Harmattan, $210 \mathrm{p}$.

Todd Emmanuel (1994) Le destin des immigrés. Assimilation et ségrégation dans les démocraties occidentales, Paris, Éditions du Seuil, 398 p.

Veith Blandine (2005) Engagement associatif et individuation de femmes migrantes, Revue Européenne des Migrations Internationales, 21 (3), pp. 53-67.

Williams Patrick (1985) Paris-New York, I'organisation de deux communautés tsiganes, L'homme, 25 (95), pp. 121-140. 


\section{Céline Bergeon}

\section{Les Roms roumains en région parisienne : les mobilisations associatives au prisme des temporalités migratoires et de l'habitat}

Cet article se propose d'étudier la présence des Roms roumains à Paris sous I'angle des manifestations culturelles. Celles-ci sont de plus en plus nombreuses dans la capitale, leurs formes et surtout leur ampleur évoluent, notamment sous l'impulsion de diverses institutions politiques ou encore associatives. "Journée mondiale des Roms ", "Roma Pride ", etc., sont des moments forts de rencontres et de visibilité culturelle qui participent réellement de la circulation et/ ou de la promotion des identités et ce, à différentes échelles (locales, nationales et internationales). Nous analyserons le paysage associatif rom en région parisienne, puis la participation des Roms roumains à ces manifestations culturelles (nature de la participation et degré d'implication) pour voir si celles-ci ont un impact direct sur les conditions de vie des Roms.

\section{Romanian Roma in Paris: Activities Associated to Migratory Temporalities and Housing}

This article studies the presence of the Roma population in Paris through the angle of cultural events. Increasingly numerous in the capital, the size and form of these events are evolving, partly due to the influence of political institutions or associations. The "World Roma Day" and the "Roma Pride" are, among others, significant events, allowing gathering opportunities and cultural visibility, which enable the circulation and/or promotion of identities at different levels (local, national and international). We will analyze the associative landscape of the Romanian Roma population and then their participation in such cultural events in order to discuss the direct impact of these events on the living conditions of the Roma population.

\section{Los gitanos rumanos en la región parisina: movilizaciones asociativas bajo el prisma de las temporalidades migratorias y de la vivienda}

En este artículo se propone estudiar la presencia de gitanos rumanos en París bajo el prisma de los eventos culturales. Este tipo de manifestaciones son cada vez más numerosas en la capital francesa, sus formas y especialmente su amplitud se encuentran en auge bajo el impulso de diferentes instituciones o asociaciones políticas. El "Día Mundial de los Romanís», la «Pride Romaní», etc., constituyen momentos importantes de encuentros y de visibilidad cultural que participan realmente en la circulación y/o en la promoción de las identidades a diferentes escalas (local, nacional, internacional). El artículo analiza las asociaciones gitanas en la región de París y, a continuación, la participación de gitanos rumanos en tales eventos culturales (naturaleza de la participación y grado de implicación) a fin de constatar si tal participación puede tener un impacto en las condiciones de vida del colectivo. 